\title{
Realization of SIMO biquad filters and quadrature sinusoidal oscillators using OTRAs
}

\author{
Garima*, Pragati Kumar and Data Ram Bhaskar \\ Department of Electrical Engineering, Delhi Technological University, Delhi, India \\ * Corresponding Author : garimasolanki@dtu.ac.in
}

\begin{abstract}
In this communication, two single-input multiple-output (SIMO) type biquad filters employing operational transresistance amplifiers (OTRAs) have been presented. Various parameters of the proposed filter circuits viz. pole frequency $(\varpi)$, pole quality factor $(\mathrm{Q})$ and the gain are independently controllable. The proposed filter configurations can also be converted into low frequency oscillators with fully uncoupled control of condition of oscillation and frequency of oscillation with quadrature output voltages. The active and passive sensitivities of the proposed circuits have been found to be small. SPICE simulation results and experimental validation of the proposed circuits employing OTRAs realized with AD844 type CFOAs have also been presented.
\end{abstract}

Keywords: SIMO biquad filters; Voltage mode; Quadrature sinusoidal oscillators; Operational transresistance amplifier.

\section{INTRODUCTION}

RC active filters find widespread applications in various low-medium frequency applications. Numerous active building blocks (ABBs) viz. operational amplifier, operational transconductance amplifier, operational transresistance amplifier, current differencing buffered amplifier, current conveyor and its various derivatives, voltage differencing buffered amplifier and voltage conveyor, etc. have been employed for designing such filters [1-15].

Of the numerous $\mathrm{ABBs}$, the OTRA has been used more in analog signal processing and generation circuits because virtual ground is present at its input terminals, which removes the effect of parasitic immittances present there. Also, its low output impedance facilitates easy cascadability. Therefore, various active filters using OTRAs have appeared in the literature. As the proposed work deals with SIMO type biquad filters realized with OTRAs, it is worthwhile to present an overview of SIMO type biquad filters using OTRA proposed previously by various authors [7-14], so that the proposed work is put in proper perspective.

Soliman and Madian [7] presented active RC and MOS-C implementation of the classical KHN biquad using OTRAs. The active RC version of the circuit uses three OTRAs, six resistors and two capacitors. In this circuit, high pass (HP), low pass (LP) and inverting band pass (BP) responses were available at three low output impedance nodes. In this a MOS-C version of the circuit is also presented where the resistors were subsituted by MOSFETs that operate in linear region and had complete non-linear cancellation. In [8], the same authors presented the MOS-C version of the classical Tow Thomas biquad circuit using two OTRAs. Two transresistance mode universal filter circuits employing OTRAs have been reported in [9]. One of these circuit is based on SIMO topology, while the other is based on MISO (multiple input single output) topology. All the five second order filter responses, namely, LP, HP, BP, band elimination 
and the all pass, are available in transresistance mode in both the circuits. Orthogonal tunability of the $₫$ and the Q is possible but the gain remains fixed. In [10], Senani, Singh, Gupta and Bhaskar have presented two OTRA and four passive resistor- based active-R realization of inverting LP and BP filter which utilizes the poles of OTRAs to replace the capacitors. All the three filter parameters of the proposed band pass filter can be controlled by changing different resistors. The reported filter circuit has been derived from an oscillator circuit simply by changing the polarities of the OTRA. But in it one terminal of the OTRA is left open, thus not fully utilising the intrinsic property of the OTRA. In [11], a signal flow graph (SFG) based approach has been used for realization of SIMO type filters employing differentiators, realized with OTRAs, resistors (R) and capacitors $(\mathrm{C})$. The circuits have been classified into two categories- one realizing the LP, inverting BP and the inverting HP transfer function in voltage mode, while the other realizing inverting LP, BP and the HP responses in voltage mode. A total of sixteen variants have been derived from the proposed differentiator based SFG. All these structures use three OTRAs, six R and two C and provide independent adjustment of the filter performance parameters. But in all these realizations one of the input terminal of the OTRA has been left open. Analytical synthesis method, which is based on the state variable method of active network synthesis, has been used to realize a multifunction biquad filter using three OTRAs, two C and six R [12]. Three filter functions, namely, the HP, inverting BP and the LP are available at the output of the three OTRAs. As the capacitors are not placed in the feedback around the OTRAs, these capacitors do not introduce additional parasitic poles in conjunction with the finite transresistance of the OTRAs. A MOS-C version of the circuit where all the $\mathrm{R}$ have been replaced by MOS resistors has also been presented. A voltage mode biquad filter employing three OTRAs, six R and two C realizing LP, BP and HP responses have been presented in [13]. No component matching constraint is required for realization of the filtering functions and the filter parameters are independently tunable. In [14], a universal filter circuit has been reported which employs five OTRAs, twelve R and two C. The pole frequency and the pole quality factors are orthogonally tunable while the gain can be tuned independently. Passive components matching constraints have been prescribed for the realization of band reject and all pass filter responses. From the above description of the reported works on SIMO type biquad filters, it thus emerges that the catalogue of the OTRA-based biquad filters is far from being complete. Therefore, the primary intent of this work is to present two new structures of SIMO type biquad filters using OTRAs which realize a LPF, BPF and HPF utilizing the intrinsic (current differencing) property of OTRA. The proposed filters have orthogonal control of the $₫$ and $\mathrm{Q}$, while the gain can be controlled independently.

Furthermore, the filter circuits can be easily modified into quadrature sinusoidal oscillators capable of generating low frequency sinusoids having fully uncoupled control of condition of oscillation (CO) and frequency of oscillation (FO) with quadrature outputs.

\section{PROPOSED SIMO TYPE BIQUAD FILTER CONFIGURATIONS}

The circuit symbol and port relations of OTRA are shown in Fig.1 and equation (1) respectively.

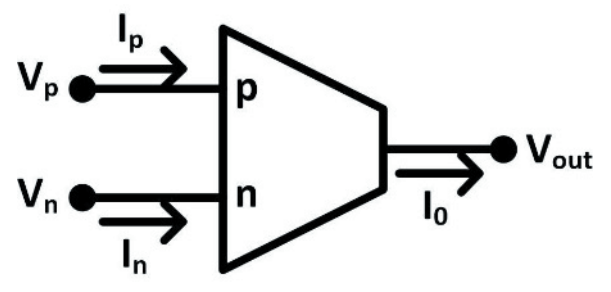

Figure 1. Symbolic representation of OTRA 


$$
\left[\begin{array}{c}
\mathrm{V}_{\mathrm{p}} \\
\mathrm{V}_{\mathrm{n}} \\
\mathrm{V}_{\text {out }}
\end{array}\right]=\left[\begin{array}{ccc}
0 & 0 & 0 \\
0 & 0 & 0 \\
\mathrm{R}_{\mathrm{m}} & -\mathrm{R}_{\mathrm{m}} & 0
\end{array}\right]\left[\begin{array}{c}
\mathrm{I}_{\mathrm{p}} \\
\mathrm{I}_{\mathrm{n}} \\
\mathrm{I}_{\mathrm{o}}
\end{array}\right]
$$

Here, $\mathrm{V}_{\text {out }}=\left(\mathrm{I}_{\mathrm{p}}-\mathrm{I}_{\mathrm{n}}\right) \mathrm{R}_{\mathrm{m}}$

Under the conditions of negative feedback and $R_{m} \rightarrow \infty$ we get $\left(I_{p}-I_{n}\right) \rightarrow 0$, This makes $I_{p}=I_{n}$. The proposed SIMO type filter configurations are presented in Fig.2.

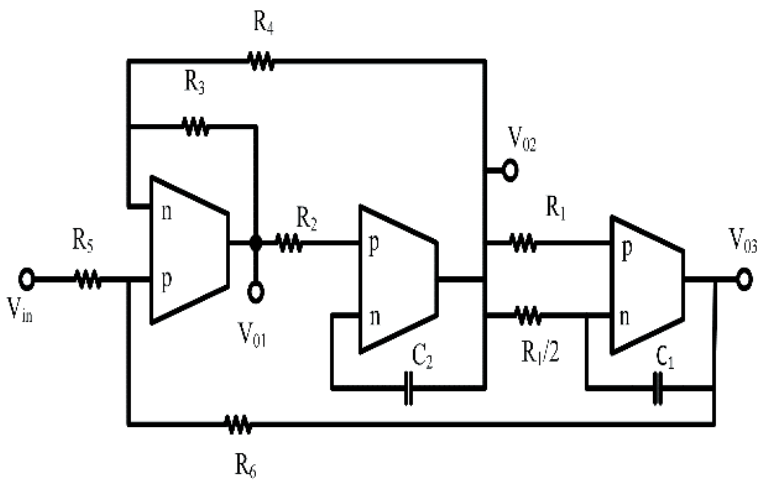

(a)



(b)

Figure 2. Proposed single input multiple output filter configurations

Analysis of the circuits given in Fig. 2(a) and 2(b), using the port relations of the ideal OTRA, gives the different transfer function of the filters namely high pass filter (HPF), band pass filter (BPF) and low pass filter (LPF) as given by equations $(2-5)$.

Fig. 2(a)

$$
\begin{gathered}
\text { BPF } \\
\frac{\mathrm{V}_{01}}{\mathrm{~V}_{\text {in }}}=\left(\frac{\mathrm{R} 3}{\mathrm{R}_{5}}\right)\left(\frac{\mathrm{s}^{2}}{\mathrm{D}(\mathrm{s})}\right) \quad \frac{\mathrm{V}_{02}}{\mathrm{~V}_{\text {in }}}=\left(\frac{\mathrm{R}_{4}}{\mathrm{R}_{5}}\right)\left(\frac{\mathrm{s}\left(\frac{\mathrm{R}_{3}}{\mathrm{C}_{2} \mathrm{R}_{2} \mathrm{R} 4}\right)}{\mathrm{D}(\mathrm{s})}\right) \quad \frac{\mathrm{V}_{03}}{\mathrm{~V}_{\text {in }}}=\left(\frac{-\mathrm{R}_{6}}{\mathrm{R} 5}\right)\left(\frac{\frac{\mathrm{R}_{3}}{\mathrm{R}_{6}}}{\frac{\mathrm{C}_{1} \mathrm{C}_{2} \mathrm{R}_{1} \mathrm{R}_{2}}{\mathrm{D}(\mathrm{s})}}\right) \\
\text { where } D(s)=s^{2}+s\left(\frac{R_{3}}{C_{2} R_{2} R_{4}}\right)+\left(\frac{\frac{R_{3}}{R_{6}}}{C_{1} C_{2} R_{1} R_{2}}\right)
\end{gathered}
$$


Fig. 2(b)

$$
\begin{aligned}
& \text { HPF } \\
& \text { BPF } \\
& \text { LPF } \\
& \frac{\mathrm{V}_{01}}{\mathrm{~V}_{\text {in }}}=\left(\frac{-\mathrm{R}_{3}}{\mathrm{R}_{5}}\right)\left(\frac{\mathrm{s}^{2}}{\mathrm{D}(\mathrm{s})}\right) \quad \frac{\mathrm{V}_{02}}{\mathrm{~V}_{\text {in }}}=\left(\frac{\mathrm{R}_{4}}{\mathrm{R}_{5}}\right)\left(\frac{\mathrm{s}\left(\frac{\mathrm{R}_{3}}{\mathrm{C}_{2} \mathrm{R}_{2} \mathrm{R}_{4}}\right)}{\mathrm{D}(\mathrm{s})}\right) \quad \frac{\mathrm{V}_{03}}{\mathrm{~V}_{\text {in }}}=\left(\frac{\mathrm{R}_{6}}{\mathrm{R}_{5}}\right)\left(\frac{\frac{\mathrm{R}_{3}}{\mathrm{R}_{6}}}{\left.\frac{\mathrm{C}_{1} \mathrm{C}_{2} \mathrm{R}_{1} \mathrm{R}_{2}}{\mathrm{D}(\mathrm{s})}\right)}\right. \\
& \text { where } D(s)=s^{2}+s\left(\frac{R_{3}}{C_{2} R_{2} R_{4}}\right)+\left(\frac{\frac{R_{3}}{R_{6}}}{C_{1} C_{2} R_{1} R_{2}}\right)
\end{aligned}
$$

The filter parameters, namely, $\omega, \mathrm{Q}$ and bandwidth (BW) can be obtained from equation (3) and (5), which are given below:

$$
\omega=\sqrt{\frac{\left(\frac{R_{3}}{R_{6}}\right)}{C_{1} C_{2} R_{1} R_{2}}} \quad Q=R_{4} \sqrt{\frac{C_{2} R_{2}}{C_{1} R_{1} R_{3} R_{6}}} \quad B W=\frac{R_{3}}{C_{2} R_{2} R_{4}}
$$

From equation (6), it may be seen that the $\mathrm{Q}$ can be varied by varying $\mathrm{R}_{4}$ without changing $\omega$. On the other hand, the $\mathrm{BW}$ can be tuned by changing the value of $\mathrm{R}_{4}$ without changing the value of $\omega$, which in turn, can be tuned without disturbing the $\mathrm{BW}$ by varying either $\mathrm{R}_{1}$ or $\mathrm{R}_{6}$.

The gain $\left(\mathrm{H}_{0}\right)$ of HPF, BPF and LPF are derived from equations (2) and (4) which are given below:

$$
H_{0(H P F)}=\frac{R_{3}}{R_{5}} \quad H_{0_{(B P F)}}=\frac{R_{4}}{R_{5}} \quad H_{0_{(L P F)}}=\frac{R_{6}}{R_{5}}
$$

It is clearly seen from equation (7) that the gain of HPF, BPF and LPF can be independently controlled simply by varying $\mathrm{R}_{5}$, without affecting $\oplus$, $\mathrm{Q}$ and $\mathrm{BW}$.

\section{SENSITIVITY ANALYSIS}

The tolerances in component values cause deviation in the response of actual filter from the ideal response. Therefore, we have evaluated the sensitivity of filter parameters $\omega, \mathrm{Q}$ and BW with R and C. These sensitivities can be calculated using the classical formula 


$$
S_{x}^{F(x)}=\frac{\partial(F(x))}{\partial x}\left(\frac{x}{F(x)}\right)
$$

Where $\mathrm{F}(\mathrm{x})$ denotes a circuit parameter and $\mathrm{x}$ denotes parameter of interest.

The sensitivities of $\omega_{0}, \mathrm{Q}_{0}$ and $\mathrm{BW}$ with respect to parameter of interest have been evaluated and tabulated in Table 1 .

Table 1. Sensitivities of filter parameters with respect to passive components

\begin{tabular}{|c|c|c|c|c|c|c|c|c|}
\hline Parameter & $\mathrm{R}_{1}$ & $\mathrm{R}_{2}$ & $\mathrm{R}_{3}$ & $\mathrm{R}_{4}$ & $\mathrm{R}_{5}$ & $\mathrm{R}_{6}$ & $\mathrm{C}_{1}$ & $\mathrm{C}_{2}$ \\
\hline $\boldsymbol{S}_{\boldsymbol{x}}^{\boldsymbol{\omega}}$ & -0.5 & -0.5 & 0.5 & 0 & 0 & -0.5 & -0.5 & -0.5 \\
\hline $\boldsymbol{S}_{\boldsymbol{x}}^{\boldsymbol{Q}}$ & -0.5 & 0.5 & -0.5 & 1 & 0 & -0.5 & -0.5 & 0.5 \\
\hline $\boldsymbol{S}_{\boldsymbol{x}}^{\boldsymbol{B} \boldsymbol{W}}$ & 0 & -1 & 1 & -1 & 0 & 0 & 0 & -1 \\
\hline
\end{tabular}

From Table 1, it is seen that the sensitivities of $₫$, Q and BW with passive components vary between \pm 1 .

\subsection{Non-Ideal Performance Analysis}

The consequence of major non-idealities immanent in the OTRA on the realized filters are considered in this section. Taking into account the single pole model for the trans-resistance gain $\mathrm{R}_{\mathrm{m}}[16]$, given below as:

$$
\mathrm{R}_{\mathrm{m}}(\mathrm{s})=\frac{\mathrm{R}_{0}}{1+\frac{\mathrm{s}}{\omega_{0}}}
$$

For high frequency applications, $\mathrm{R}_{\mathrm{m}}(\mathrm{s})$ reduces to

$$
\mathrm{R}_{\mathrm{m}}(\mathrm{s}) \approx \frac{1}{\mathrm{sC}_{\mathrm{p}}} \text { where } \mathrm{C}_{\mathrm{p}}=\frac{1}{\mathrm{R}_{0} \omega_{0}}
$$

Using this non-ideal model of the OTRA, the non-ideal transfer functions of the filters have been determined as:

$$
\frac{V_{03}}{V_{i n}}=-\frac{R_{6}}{R_{5}}\left(\frac{R_{3} R_{4}}{D_{N I}}\right)
$$




$$
\begin{gathered}
\frac{V_{02}}{V_{\text {in }}}=\frac{R_{4}}{R_{5}}\left\{\frac{R_{1} R_{3} R_{6}\left(C_{1}+C_{P}\right)}{D_{N I}}\right\} \\
\frac{V_{01}}{V_{\text {in }}}=\frac{R_{3}}{R_{5}}\left\{\frac{s^{2} R_{1} R_{2} R_{4} R_{6}\left(C_{1}+C_{P}\right)\left(C_{2}+C_{P}\right)}{D_{N I}}\right\} \\
D_{N I}(s)=s^{3} C_{P} R_{1} R_{2} R_{4} R_{6}\left\{\left(C_{1}+C_{P}\right)\left(C_{2}+C_{p}\right)\right\}+s^{2} R_{1} R_{2} R_{4} R_{6}\left\{\left(C_{1}+C_{p}\right)\left(C_{2}+C_{p}\right)\right\} \\
+s R_{1} R_{3} R_{6}\left(C_{1}+C_{P}\right)+R_{3} R_{4}
\end{gathered}
$$

It is noted from equation (10) that for large values of $\mathrm{R}_{\mathrm{o}}$ (ideally, infinite), the value of $\mathrm{C}_{\mathrm{p}}$ will be very small, we may neglect the cubic term in the expression of $\mathrm{D}_{\mathrm{NI}}(\mathrm{s})$. Thus, equation (14) now reduces to

$$
D_{N I}(s)=s^{2} R_{1} R_{2} R_{4} R_{6}\left\{\left(C_{1}+C_{P}\right)\left(C_{2}+C_{p}\right)\right\}+s R_{1} R_{3} R_{6}\left(C_{1}+C_{P}\right)+R_{3} R_{4}
$$

From equation (15), we can derive expressions for non-ideal pole frequency, quality factor and bandwidth. These expressions are given by equation (16), (17) and (18) respectively.

$$
\begin{gathered}
\omega_{N I}=\omega\left\{1+\frac{C_{P}}{C_{1} C_{2}}\left(C_{1}+C_{2}+C_{P}\right)\right\}^{-\frac{1}{2}} \\
Q_{N I}=Q\left\{1+\frac{C_{P}}{C_{1} C_{2}}\left(C_{1}+C_{2}+C_{P}\right)\right\}^{\frac{1}{2}} \\
B W_{N I}=B W \frac{1+\frac{C_{P}}{C_{1}}}{\left\{1+\frac{C_{P}}{C_{1} C_{2}}\left(C_{1}+C_{2}+C_{P}\right)\right\}^{\frac{1}{2}}}
\end{gathered}
$$

It is thus observed that the non-ideal values of the filter parameters deviate little from their ideal values as $\mathrm{C}_{\mathrm{P}}$ is very small.

\section{PROPOSED VM FULLY UNCOUPLED QUADRATURE SINUSOIDDAL OSCILLATOR}

A careful look at the transfer functions given by equations (2) and (4) reveals that the bandpass filter outputs $\left(\mathrm{V}_{02}\right)$ available in both the circuits shown in Fig. 2 are non-inverting. Thus, these filters can be modified into second order 
sinusoidal oscillators by simply closing the loop between the input terminals (by removing the external input) and the terminal at which the voltage $\mathrm{V}_{02}$ are available. The circuit diagrams of oscillators are shown below in Fig. 3.



(a)

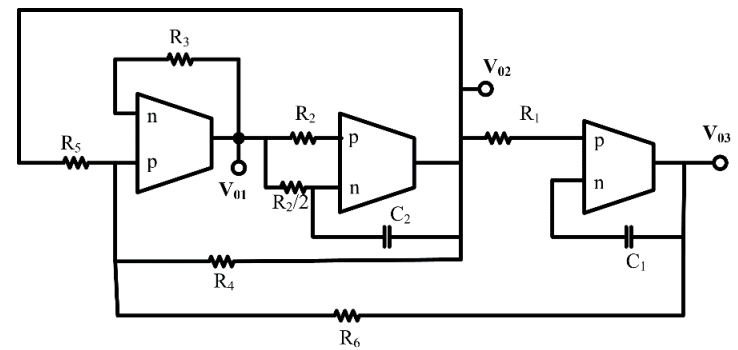

(b)

Figure 3. Proposed quadrature sinusoidal oscillators

Assuming ideal OTRAs, routine circuit analysis of Fig. 3 (a and b) gives the characteristic equation as:

$$
s^{2}+\left(\frac{R_{3}}{C_{2} R_{2}}\right)\left(\frac{1}{R_{4}}-\frac{1}{R_{5}}\right) s+\frac{R_{3}}{C_{1} C_{2} R_{1} R_{2} R_{6}}=0
$$

The $\mathrm{CO}$ and $\mathrm{FO}$ of the proposed oscillators are given as:

$$
C O: R_{5}-R_{4} \leq 0
$$

$$
F O: \omega_{0}=\sqrt{\frac{\left(\frac{R_{3}}{R_{6}}\right)}{C_{1} C_{2} R_{1} R_{2}}}
$$

From equation (21), it may be noted out that the proposed oscillator circuits have fully uncoupled control of $\mathrm{CO}$ and FO, i.e., $\mathrm{CO}$ can be independently set through two resistors $\mathrm{R}_{4}$ and $\mathrm{R}_{5}$ without affecting $\mathrm{FO}$ which has four degree of freedom to tune independently (using resistors $\mathrm{R}_{1}, \mathrm{R}_{2}, \mathrm{R}_{3}$ and $\mathrm{R}_{6}$ ) and also it can be tuned through capacitors $\mathrm{C}_{1}$ and $\mathrm{C}_{2}$.

From the equation of FO, it may be noted that the proposed oscillator has a feature to generate low frequency oscillations which may be done if the ratio of the resistors $R_{3}$ and $R_{6}$ is selected to be very small by choosing $R_{6}$ much larger than $\mathrm{R}_{3}$

For the circuits of oscillator presented in Fig.3, two quadrature voltages are available, first is between $\mathrm{V}_{01}$ and $\mathrm{V}_{02}$ and the second, between $\mathrm{V}_{02}$ and $\mathrm{V}_{03}$, as can be seen from equation (22) and (23).

$$
\begin{array}{cc}
\frac{V_{02}}{V_{01}}(s)=\frac{1}{\mathrm{sC}_{2} \mathrm{R}_{2}} & \frac{V_{03}}{V_{02}}(s)=\frac{1}{\mathrm{sC}_{1} \mathrm{R}_{1}} \\
\frac{V_{02}}{V_{01}}(j \omega)=\frac{1}{\omega \mathrm{C}_{2} \mathrm{R}_{2}} e^{-90^{\circ}} & \frac{V_{03}}{V_{02}}(j \omega)=\frac{1}{\omega \mathrm{C}_{1} \mathrm{R}_{1}} e^{-90^{\circ}}
\end{array}
$$




\section{FREQUENCY STABILITY}

For an oscillator, frequency stability is an essential figure of merit. It measures the capability of an oscillator to maintain its fundamental frequency constant for a long period. The frequency stability $\mathrm{S}^{\mathrm{F}}$ is defined as: where and is the phase of the open loop transfer function of an oscillator.

We have used the open loop transfer function of BPF given in equation (2) to calculate the frequency stability factor and found it to be -2n, when $R_{1}=R_{2}=R_{3}=R_{6}=R / n, R_{4}=R_{5}=R$, and $C_{1}=C_{2}=C$. The calculated $S^{F}$ shows that the FO has a very high frequency stability, when the value of $\mathrm{n}$ is made very large.

\section{SIMULATION RESULTS AND EXPERIMENTAL VERIFICATION}

Validation of the proposed filters and oscillator circuits has been done through simulation using PSPICE macro model of AD844 and the same have been verified through experimental results also. A discrete implementation of the OTRA using two current feedback operational amplifiers (CFOAs) ICs, AD 844 [13] as shown in Fig. 4, was used in SPICE simulations as well as experimental verification of the results. The power supplies used were $\pm 5 \mathrm{~V}$. In the following, we present the simulation and experimental results for the filter circuit shown in Fig. 2 (a) and the sinusoidal oscillator circuit shown in Fig. 3(a)

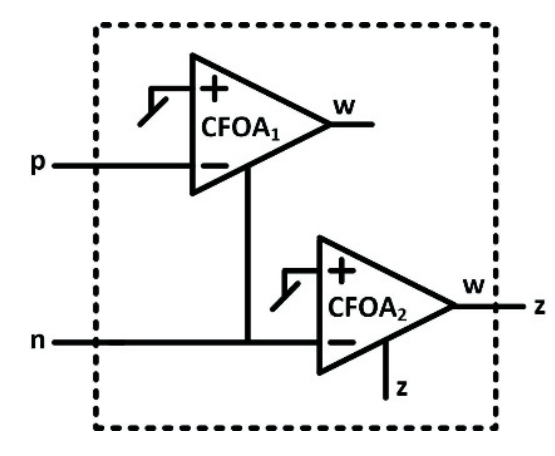

Figure 4. CFOA implementation of OTRA

The filters were designed for a nominal $\boldsymbol{\omega}_{0}$ of $15.9 \mathrm{kHz}$, Q of 1, BW (for BPF) $=15.9 \mathrm{kHz}$ and gain 1 . The passive components used were: $\mathrm{R}_{\mathrm{i}}=10 \mathrm{k} \Omega$ (where $\mathrm{i}=1$ to 6 ) and $\mathrm{C}_{1}=\mathrm{C}_{2}=1 \mathrm{nF}$.

The experimental frequency responses of filters obtained on Keysight DSOX3034T superimposed on the simulated frequency response obtained using PSPICE, have been shown in Fig. 5. The time response of these filters have also been depicted in Fig.6 where input voltages of $1 \mathrm{~V}_{\mathrm{pp}}$ at $15.9 \mathrm{kHz}$ were applied.

Tunability of Q: We have also demonstrated the tunability of $\mathrm{Q}$ without changing the value of $\omega_{0}$, and the gain by varying $\mathrm{R}_{4}$ and $\mathrm{R}_{5}$ simultaneously. We have taken $\mathrm{R}_{4}=\mathrm{R}_{5}=1 \mathrm{k} \Omega, 4.7 \mathrm{k} \Omega, 10 \mathrm{k} \Omega$ and $22 \mathrm{k} \Omega$ for $\mathrm{Q}=0.1,0.47,1$ and 2.2 respectively. For these values $\omega_{0}=15.9 \mathrm{kHz}$ and gain $=1$. The respective graphs have been shown in Fig. 7(a).

Tunability of $\omega_{0}$ : From the expression of the pole frequency, it may be noted that the pole frequency can be varied without changing the values of the BW and the gain by varying the value of $R_{6}$. In Fig. 7(b), we have presented the variation of $\omega_{0}$ for a given value of BW and gain by varying $\mathrm{R}_{6}(4.7 \mathrm{k} \Omega, 10 \mathrm{k} \Omega, 15 \mathrm{k} \Omega$ and $22 \mathrm{k} \Omega$ ) for value of pole frequency as $23.19 \mathrm{kHz}, 15.9 \mathrm{kHz}, 12.98 \mathrm{kHz}$, and $10.72 \mathrm{kHz}$ respectively. In Fig. 7(c) we have depicted the variation of gain for a given value of pole frequency and bandwidth. 




Figure 5. Simulated (Sim) and experimental (Exp) frequency responses of proposed filters

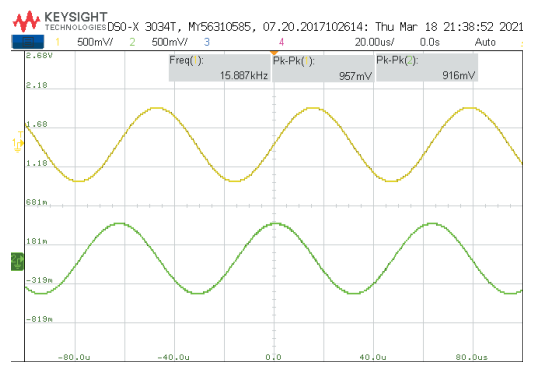

(a)

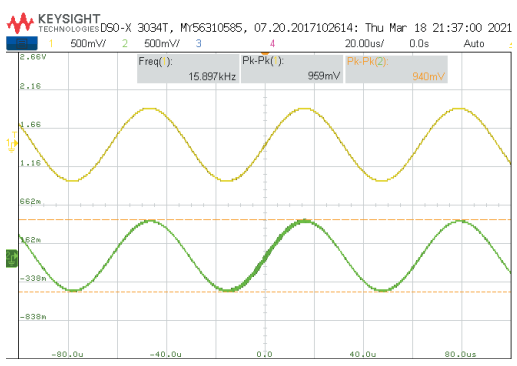

(b)

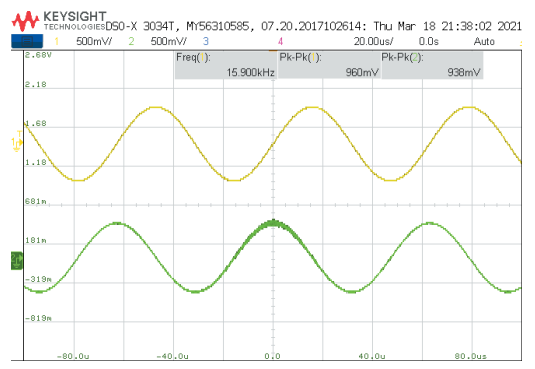

(c)

Figure 6. Transient input and output voltages of (a) HPF (b) BPF and (c) LPF

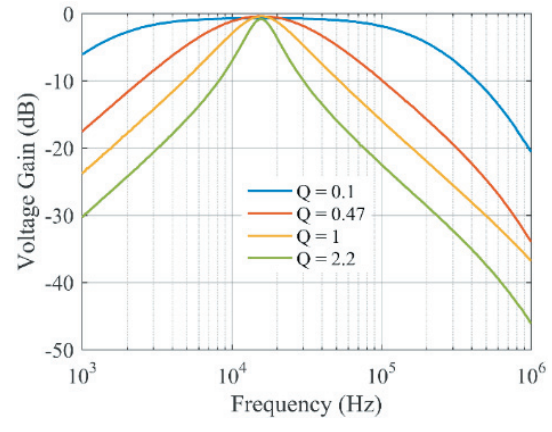

(a)

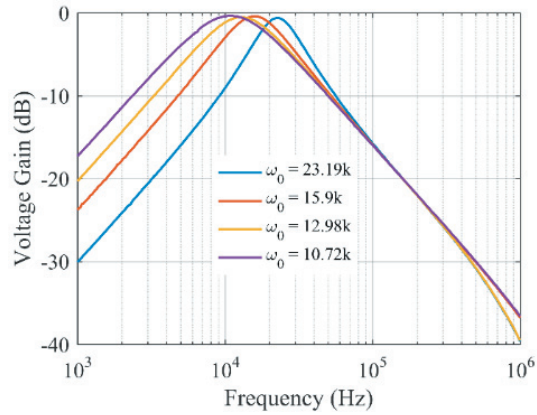

(b)

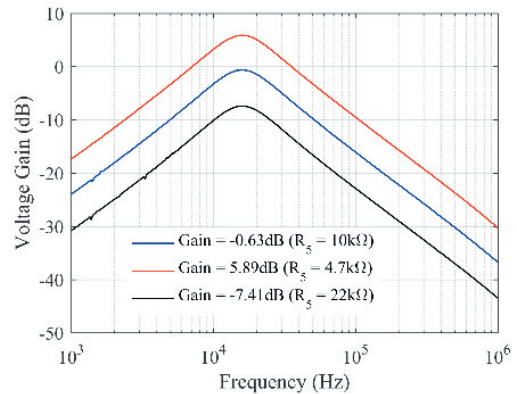

(c)

Figure 7. (a) Tunability of Q with $\omega_{0}$ (b) Tunability of $\omega_{0}$ (c) Tunability of Gain

\section{QUADRATURE SINUSOIDAL OSCILLATOR}

The quadrature sinusoidal oscillator circuits presented in Fig. 3(a) was bread-boarded with the same values of passive components which were used for filter design. Two quadrature output voltage responses $\left(\mathrm{V}_{01}, \mathrm{~V}_{02}\right)$ and $\left(\mathrm{V}_{02}\right.$ and $\mathrm{V}_{03}$ ), Lissajous patterns and frequency spectrums have been displayed in Fig. 8 and Fig. 9 respectively. The effect 
of varying resistance $R_{2}$ on frequency is shown in Fig. 9(d). Fig. 10(a) shows that for different values of $R_{4}$ and $R_{5}$ the variation in frequency with $\mathrm{R}_{2}$ remains the same, thus, confirming the decoupled nature of tuning of FO and $\mathrm{CO}$. Variation of THD and peak to peak voltage outputs $\mathrm{V}_{01}, \mathrm{~V}_{02}$ and $\mathrm{V}_{03}$ with frequency has been shown in Fig.10(b) and Fig. 10(c) respectively. Similar simulation and experimental results were obtained for the circuits of Fig. 2(b) and 3(b) but they have not been explicitly shown here to conserve space.

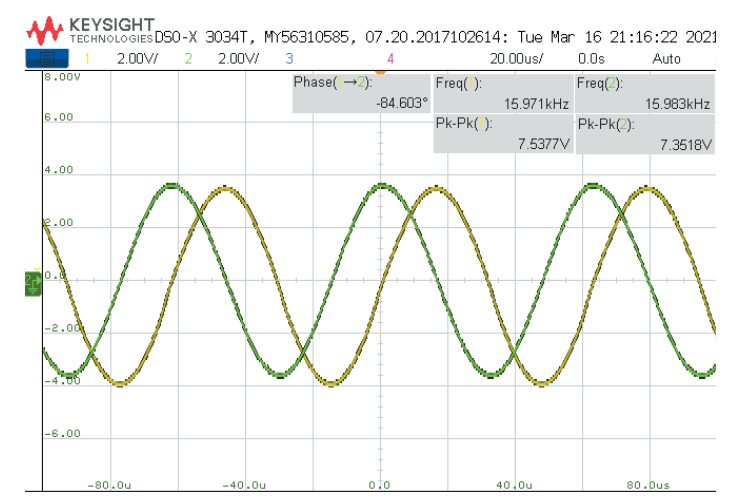

(a)

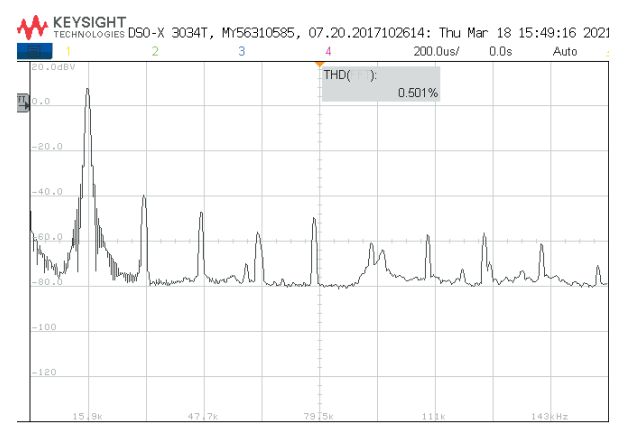

(c)



(b)

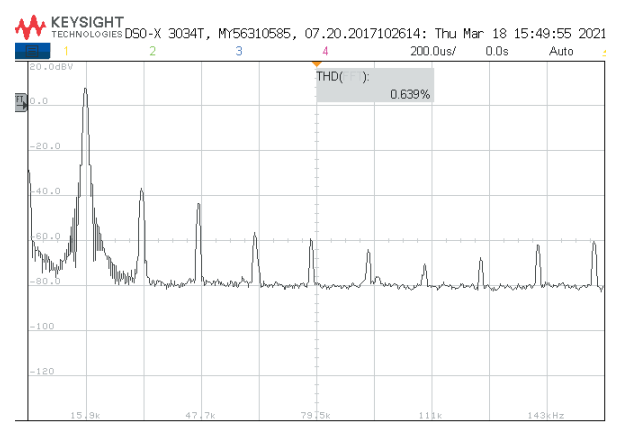

(d)

Figure 8 (a) Experimental transient responses between quadrature voltage $\mathrm{V}_{01}$ and $\mathrm{V}_{02}$ (b) Lissajous pattern (c) frequency spectrum of voltage $V_{01}$ (d) Frequency spectrum of voltage $V_{02}$

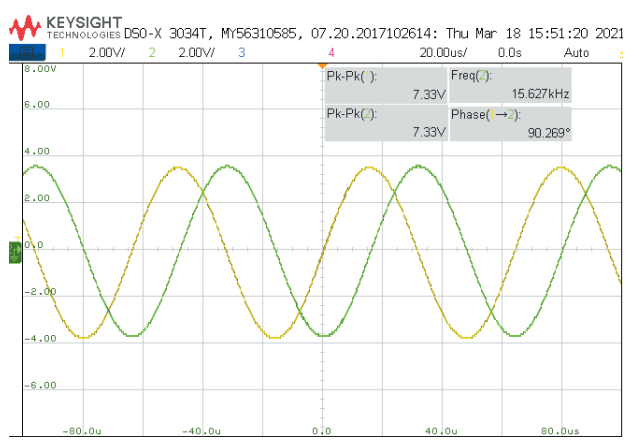

(a)

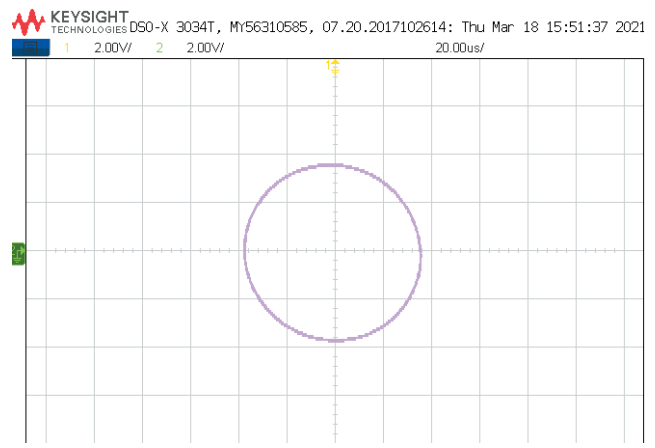

(b) 




(c)



(d)

Figure 9 (a) Experimental transient responses between quadrature voltage $\mathrm{V}_{02}$ and $\mathrm{V}_{03}$ (b) Lissajous pattern (c) Frequency spectrum of voltage $\mathrm{V}_{03}$ (d) Variation in frequency with resistance $\mathrm{R}_{2}$

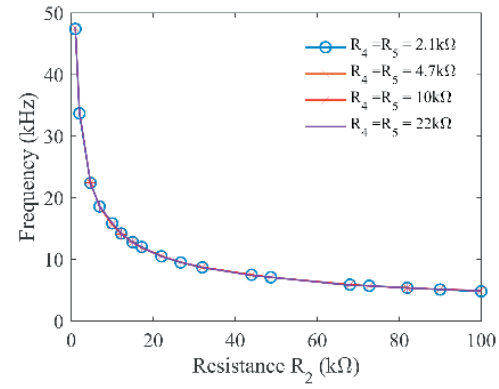

(a)

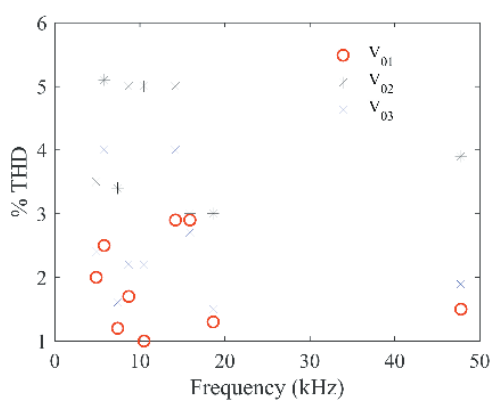

(b)

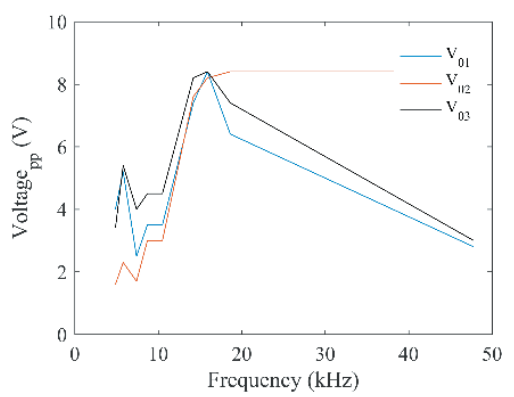

(c)

Figure 10 (a) CO decoupled tuning of FO (b)Variation in THD with frequency (c)Variation in peak to peak voltage with frequency.

\section{CONCLUDING REMARKS}

In this communication, we have presented SIMO type biquad filter structures employing three OTRAs, two capacitors and seven resistors. The presented filter configurations are capable of providing three different filter functions at low output impedance terminals. The proposed filter configurations have orthogonal tunability of $₫$ and Q. Also, its $₫$ can be independently tuned without changing the BW through separate resistors. Finally, the gain of the different filters can also be varied without changing $₫$ and Q. Furthermore, the filter circuits can be modified into oscillator circuits, capable of low frequency oscillations, with fully decoupled control of CO and FO. The passive sensitivities of $₫$, Q and BW have been evaluated and found to be low. The frequency stability factors of the proposed oscillators have also been studied and found to be large when ' $n$ ' becomes larger. Feasibleness of the proposed filters and oscillator circuits has been validated using PSPICE and experimental results. It is hoped that the proposed circuits will enrich the existing repertoire of the existing SIMO type biquad filters realized with OTRAs. 


\section{REFERENCES}

Wang, San-Fu, Hua-Pin Chen, Yitsen Ku, and Yi-Chun Lin. 2019. Versatile tunable voltage-mode biquadratic filter and its application in quadrature oscillator. Sensors 19 (10) : 2349.

Wang, S.F., Chen, H.P., Ku, Y. and Chen, P.Y. 2019. A CFOA-based voltage-mode multifunction biquadratic filter and a quadrature oscillator using the CFOA-based biquadratic filter. Applied Sciences, 9(11), p.2304.

Chang, Chun-Ming. 1997. Multifunction biquadratic filters using current conveyors. IEEE Transactions on Circuits and Systems II: Analog and Digital Signal Processing 44 (11): 956-958.

Bashir, Sheikh Ajaz, and Nisar Ahmed Shah. 2012. Voltage mode universal filter using current differencing buffered amplifier as an active device.

Kacar, Furat, Abdullah Yesil, and Abbas Noori. 2012. New CMOS realization of voltage differencing buffered amplifier and its biquad filter applications. Radioengineering 1) Yl) : 333-339.

Koton, Jaroslav, Norbert Herencsár, and Kamil Vrba. 2011. KHN-equivalent voltage-mode filters using universal voltage conveyors. AEU-International Journal of Electronics and Communications 65 (2): 154-160.

Soliman, Ahmed M., and Ahmed H. Madian. 2009. MOS-C KHN filter using voltage op amp, CFOA, OTRA and DCVC. Journal of Circuits, Systems, and Computers 18, (4): 733-769.

Soliman, Ahmed M., and Ahmed H. Madian. 2009. Mos-C Tow-Thomas filter using voltage OP AMP, current feedback OP AMP and operational transresistance amplifier. Journal of Circuits, Systems, and Computers 18 (1): 151-179.

Chadha, Ujjwal, and Tajinder Singh Arora. 2016. SIMO and MISO universal filters employing OTRA. In Communication and Computing Systems: Proceedings of the International Conference on Communication and Computing Systems.

Senani, Raj, Abdhesh Kumar Singh, Ashish Gupta, and Data Ram Bhaskar. 2016. Simple simulated inductor, low-pass/band-pass filter and sinusoidal oscillator using OTRA. Circuits and systems 7(3): 83.

Pandey, Neeta, Rajeshwari Pandey, Rashika Anurag, and Ritu Vijay. 2020. A Class of Differentiator-Based Multifunction Biquad Filters Using OTRAs. Advances in Electrical and Electronic Engineering 18 (1): 31-40.

Chang, Chun-Ming, Ying-Tsai Lin, Chih-Kuei Hsu, Chun-Li Hou, and Jiun-Wei Horng. 2011. Generation of voltage-mode OTRA-based multifunction biquad filter. Recent Researches in Instrumentation, Measurement, Circuits and Systems: 21-27.

Kumngern, Montree, Somyot Junnapiya, and Jirasak Chanwutitum. 2013. Voltage-mode lowpass, highpass, bandpass biquadratic filter using OTRAs. In 2013 IEEE International Conference on Control System, Computing and Engineering: 230-233.

Pandey, Rajeshwari, Neeta Pandey, Sajal Kumar Paul, Ajay Singh, Balamurali Sriram, and Kaushalendra Trivedi. 2012. Voltage mode OTRA MOS-C single input multi output biquadratic universal filter. Advances in Electrical and Electronic Engineering 10 (5): 337-344.

Singh, Abdhesh K., Raj Senani, and Ashish Gupta. 2018. OTRA, its implementations and applications: a state-ofthe-art review. Analog Integrated Circuits and Signal Processing 97 (2): 281-311.

Sánchez-López, C., E. Martínez-Romero, and E. Tlelo-Cuautle. 2011. Symbolic analysis of OTRAs-based circuits. Journal of applied research and technology 9 (1): 69-80. 